\title{
Model-Driven Design of Web Applications with Client-Side Adaptation
}

\author{
Stefano Ceri ${ }^{1}$, Peter Dolog ${ }^{2}$, Maristella Matera ${ }^{1}$, and Wolfgang Nejdl ${ }^{2}$ \\ 1 Dipartimento di Elettronica e Informazione - Politecnico di Milano \\ Via Ponzio, 34/5, 20133 Milano, Italy \\ \{ceri, matera\}@elet.polimi.it \\ 2 Learning Lab Lower Saxony - University of Hannover, \\ Expo Plaza 1, 30539 Hannover, Germany, \\ $\{$ dolog, nejdl\}@learninglab.de
}

\begin{abstract}
In this paper, we integrate WebML, a high-level model and technology for building server-side Web applications, with UML-Guide, a UML-based system that generates client-side guides for the adaptation of Web applications. The combination of the two systems is shown at work on an e-learning scenario: WebML is the basis of the specification of a generic e-learning system, collecting a large number of learning objects, while UML-Guide is used for building company-specific e-learning curricula. The resulting system can be considered an "adaptive hypermedia generator" in full strength, whose potential expressive power goes beyond the experiments reported in this paper.
\end{abstract}

Keywords: Personalization, UML, WebML Modeling, Web Engineering.

\section{Introduction}

In recent years, the control of Web applications has moved from the client to the server side, leading to more economical, structured, and well engineered solutions. In particular, the model-driven approach, as advocated in [3,611,17] has proved very effective in extending the classical methods and best practices of Software Engineering to the Web. Design methods now concentrate on content, navigation, and presentation design, which are orthogonally developed by means of specialized abstractions and techniques.

While server-side solutions are dominant, yet bringing some intelligence to the client may be highly beneficial in some cases [1618]. Client-side solutions can reveal as being more dynamic, more adaptive, and protective for sensitive user data. They may be very effective for "remembering" the local context or being aware of the local peculiarities of the interaction. Also, a clear separation of concerns between the client and the server may lead to interesting business opportunities and models.

This paper explores the combination of two existing approaches to the engineering of Web applications. We use the WebML method [3] and its development support environment [4] for generating the application server-side "backbone". We then integrate such a backbone with UML-Guide [9], a client-side personalization engine that dynamically generates additional interfaces and user guides for personalizing the application's fruition, by managing user profiles and context-sensitive data at client side. 
The proposed approach capitalizes on the use of two systems that both start from highlevel abstractions, and are both capable of automatic deployment of the implementations:

- The WebML method is based on the use of high-level concepts, such as the notions of entity and relationship to denote content, and of page, unit, and link to denote hypertexts. These abstractions are automatically turned into implementation artifacts by means of WebRatio, a tool for the automatic deployment of Web applications [3].

- UML-Guide is based on the use of UML state diagrams, whose nodes and arcsrepresenting states and transitions_-are turned into XMI specifications. A client-side translator, written in XSL, turns such specifications into a user interface facilitating the adaptive use of the application [9].

Coupling WebML and UML-Guide yields the following advantages:

- The use of high-level WebML abstractions in the context of UML-Guide enables the specification of a powerful client-side personalization engine. The resulting application generator can be considered an "adaptive hypermedia generator" in full strength, whose potential expressive power goes well beyond the experiment reported in this paper.

- The tools prove to be highly complementary and easily integrated, as it is sufficient to reuse concepts of WebML inside UML-Guide to provide concept interoperability, and the URL generation technique of the WebML runtime inside the UML-Guide XSL code to provide systems interoperability.

- The use of UML-driven methods in conjunction with WebML is by itself a very interesting direction of research, aiming at the integration of UML, the most consolidated software engineering method (and related technology), with WebML as a representative case of new, hypertext-specific models and techniques.

\subsection{Driving Scenario}

In order to exemplify the integration of the two methods, we refer to an e-learning scenario, in which a courseware company develops and distributes a vertical application for e-learning, running on the company's server, specified and developed through WebML1 1 . The vertical incorporates learning objects in the format of lessons, exercises, tests, definitions and examples for computer science, arranged according to the ACM categories 2 , and learning paths with checkpoints for the learner. Thus, such a vertical has learning objects as content, and navigation mechanisms, such as guided tours or indexed accesses to pages based on broad categories, enabling a generic user to access such a content though predefined navigation paths.

The vertical is used by Small-Medium Enterprises (SMEs) wishing to build personalized e-learning curricula, to be used by their employees for focused training activities. We assume that each SME has a clear instruction goal (for example, teaching its employees how to integrate Java programming into Oracle 9i), and that it can use UML-Guide

\footnotetext{
${ }^{1}$ This scenario is suggested by the ProLearn Network of Excellence, whose main focus is the enhancement of professional e-learning methods and technology; see

http://www.prolearn-project.org

2 See http://www.acm.org/class/1998/
} 
to specify it in UML; we assume that UML state diagrams, together with a vocabulary listing all the learning objects available in the vertical, may be an easy-to-use interface for the SME designer. UML-Guide specifications select the concepts to be covered in the learning paths, as well as the workflow driving the student in the learning process. We also assume that each SME has a clear view of its employees' competencies, and thus is able to constrain possibilities in the learning paths by adaptation rules based on such competencies. These rules enable adaptive content selection from the WebML vertical and also enable to adaptively indicate, show, and hide links in the learning path, and adaptively customize their targets.

\subsection{Paper Organization}

The paper is organized as follows. Section 2 introduces the WebML component, by providing an overview of the WebML method and the WebML-based specification of the vertical e-learning application. Section 3 introduces the UML-Guide method, and the specification of the client-side personalization for the vertical e-learning. Section 4 illustrates the integration of the two methods by means of an architecture where the application server-side code is generated through WebML, while personalization with respect to specific learning goals is managed at client-side through UML-Guide. Section 5 then describes the user interface generated for the integrated application. Sections 6 and 7 illustrate some related work and draw our conclusions and future work.

\section{WebML Component}

WebML is a visual language for specifying the content structure of a Web application and the organization and presentation of contents in one or more hypertexts [3]. The design process based on WebML starts with the specification of a data schema, expressing the organization of contents by means of the Entity-Relationship primitives. The WebML Hypertext Model allows then describing how contents, previously specified in the data schema, are published into the application hypertext.

The overall structure of the hypertext is defined in terms of site views, areas, pages and content units. A site view is a hypertext, designed to address a specific set of requirements. It is composed of areas, which are the main sections of the hypertext, and comprise recursively other sub-areas or pages. Pages are the actual containers of information delivered to the user; they are made of content units that are elementary pieces of information, extracted from the data sources by means of queries and published within pages. In particular, as described in Table 1 content units denote alternative ways for displaying one or more entity instances.

Their specification requires the definition of a source (the name of the entity from which the unit's content is extracted) and a selector (a condition, used for retrieving the actual objects of the source entity that contribute to the unit's content).

Within site views, links interconnect content units and pages in a variety of configurations yielding to composite navigation mechanisms. Besides representing user navigation, links between units also specify the transportation of some information (called context) that the destination unit uses for selecting the data instances to be displayed. 
Table 1. Some basic WebML content units. The whole set of units is described in [3].

\begin{tabular}{|c|c|c|}
\hline Unit name & Visual Notation & Description \\
\hline Data unit & $\underbrace{1}_{\substack{\text { Entity } \\
\text { [Selector] }}}$ & $\begin{array}{l}\text { It displays a set of attributes for a single entity in- } \\
\text { stance. }\end{array}$ \\
\hline Multidata unit & $\frac{\bigoplus}{\substack{\text { Multidata unit } \\
\text { Entity } \\
\text { [Selector] }}}$ & It displays a set of instances for a given entity. \\
\hline Index unit & 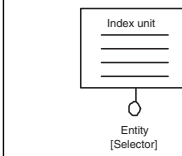 & $\begin{array}{l}\text { It displays a list of properties, also called descriptive } \\
\text { keys, of a given set of entity instances. }\end{array}$ \\
\hline Hierarchical Index unit & 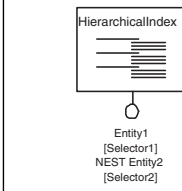 & $\begin{array}{l}\text { A variant of the index unit, which displays list of } \\
\text { properties of instances selected from multiple enti- } \\
\text { ties, nested in multi-level three. }\end{array}$ \\
\hline Scroller unit & 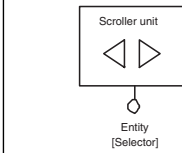 & $\begin{array}{l}\text { It represents a scrolling mechanism, } \\
\text { based on a block factor, for the elements } \\
\text { in a set of instances. }\end{array}$ \\
\hline
\end{tabular}

WebML-based development is supported by a CASE tool [4], which offers a visual environment for drawing the WebML conceptual schemas, and then supports the automatic generation of server-side code. The generated applications run in a standard runtime framework on top of Java 2 application servers, and have a flexible, service-based architecture allowing components customization.

\subsection{WebML Specification for the Vertical E-learning}

The data schema of the vertical e-learning application is centered on the concept of learning object. As reported in Figure 1 the LO entity represents descriptions of learning objects, by means of attributes inspired by the LOM standard 3 . Among them, the attribute type expresses the different types of LOs (e.g., lectures, lecture modules, definitions, exercises, tests) published by the vertical application. Each LO has associations with other LOs: for example, a lecture module can be associated with some related definitions, exercises, examples, or tests. The entity Content then represents the contents (texts, images, files) LOs consists of. In order to facilitate LO access, the schema also includes the entity Category: it stores the ACM categories that classify the LOs published by the e-learning application.

\footnotetext{
${ }^{3}$ http://ltsc.ieee.org/
} 


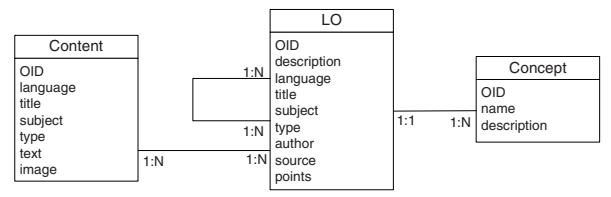

Fig. 1. WebML Data schema for the vertical e-learning application.

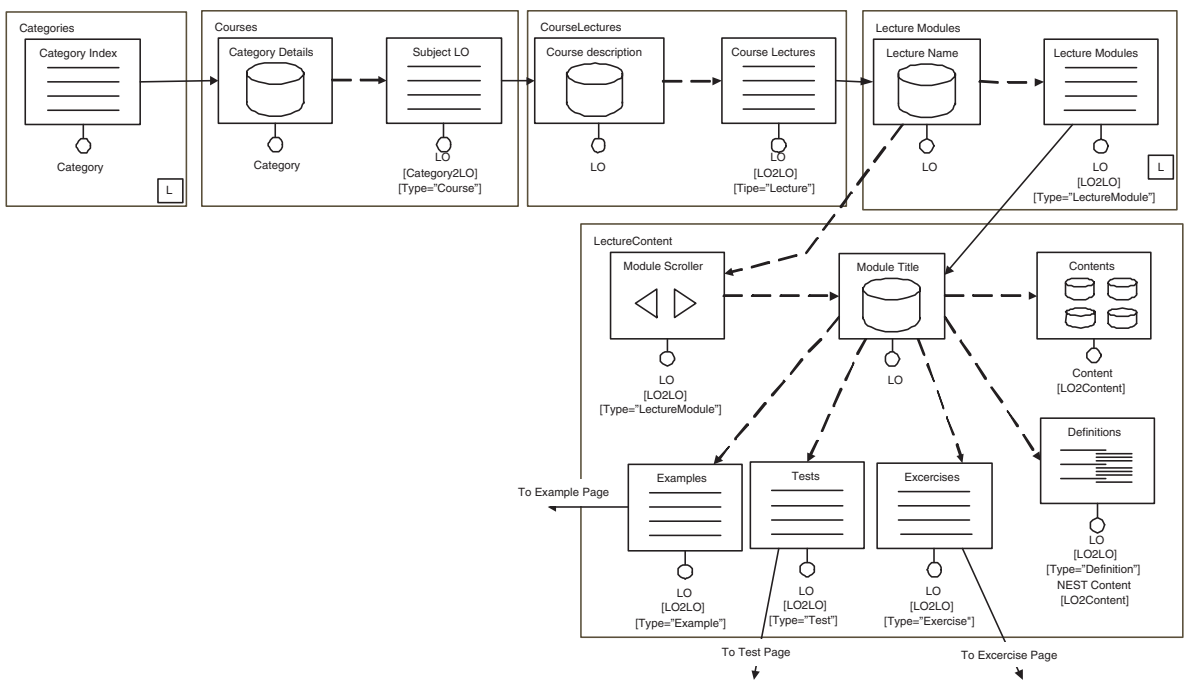

Fig. 2. The WebML specification of the hypertext interface for the vertical e-learning application.

Figure 2 reports a simplified excerpt of the WebML hypertext schema defined for the vertical e-learning application; it refers to pages for selecting a lecture module, and accessing its contents as well as associated definitions, exercises examples, and tests. The lecture module selection is operated by means of a navigation chain, in which users progressively select a subject category (Categories page), then a course that refers to the selected category (Courses page), then a lecture (CourseLectures page), and finally the lecture module they are interested in (LectureModules page). Pages Categories and LectureModules are marked with an "L" label, which indicates that they are landmark pages. This property represents that the two pages will be reachable from any other page of the hypertext, by means of landmark links.

Contents of the selected lecture module are shown in page LectureContent. As represented by the Module Scroller unit, users can browse lecture modules in a Guided Tour navigation that allows moving forward and backward in the (ordered) set of modules available for the currently selected lecture. For each module, the data unit Module Title shows the title and a short description of the learning object, the Contents multidata unit shows texts and images that compose the module, while the Definitions hierarchical index shows titles of the definitions associated with the module and, nested, the corresponding contents. Three index units then show the lists of examples, tests and 
exercises available for the current lecture module. The selection of one item from such lists leads users in a different page where the corresponding contents are displayed.

The presentation of page LectureContent, as generated by the WebML code generator, can be seen in the right frame of the Web page depicted in Figure 7

\section{UML-Guide Component}

\subsection{UML-Guide Overview}

UML State diagrams [12] are used in UML-Guide for modelling the user navigation in a hypertext. Each state represents the production of a given information chunk on the device observed by a user, and each state transition represents an event caused by user interaction that leads to the production of a new chunk of information. State diagrams therefore provide an abstraction of hypertext trails, where each trail can be adapted by taking into account the user background, level of knowledge, preferences and so on [9]. In this way, UML state diagrams are a suitable interface for UML-Guide, whose primary purpose is to build adaptive hypermedia systems.

Atomic states can be grouped into superstates. States usually refer to concepts of an application domain; thus, they can correspond to the representation of WebML pages or page units, which enable the viewing of the information entities within the WebML data schema.

Parallel substates represent information chunks to be presented simultaneously. Fork and join pseudostates are used respectively for splitting and joining computations and enabling parallelism. The SyncState pseudostate is used for synchronizing substates of parallel regions.

Transitions represent active interconnections between information chunks, and usually correspond to associations in the application domain model (thus, they can correspond to WebML links, that interconnect pages and units, and in turn depend upon the relationships of the WebML data model). Events raise transitions in a state machine; they include user-generated or system-generated events, and the latter include time events. Guards can be used to constrain transitions by adaptation rules. Usually, they consist of a predicate over user profile attributes or context information.

Actions can be performed after a transition is raised and before entering a state. Also, transitions can be associated with side effect actions, whose effect is, for example, the modification of a user profile, or the choice of presentation styles for a given chunk of information. Actions can also process parameters used in guards of outgoing part of branches. Side effect actions, as well as adaptation rules, can be assigned to entry, exit, and $d o$ actions of states.

Tagged values are domain-specific properties used to extend the semantics of elements in UML diagrams. These values can refer, for example, to concepts of the structural model of the application domain, or to specific terminologies which might be useful to identify additional navigation requirements. We will make extensive use of tagged values for linking UML diagrams of UML-Guide to WebML concepts, as illustrated in Section 4. 


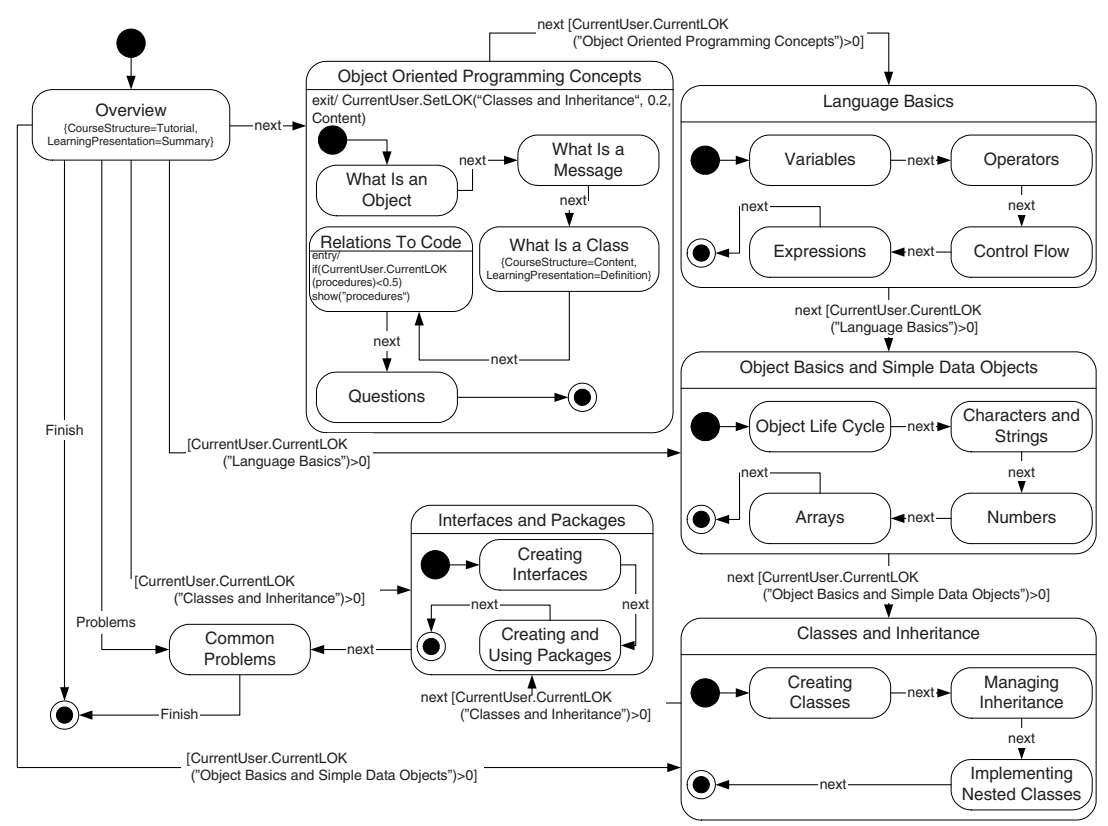

Fig. 3. A navigation model for a Java tutorial in the UML state diagram notation.

\subsection{UML-Guide State Diagram for E-learning}

The UML-Guide state diagram of Figure 3 illustrates a personalized learning environment for teaching object-oriented programming in JAVA, borrowed from a well-known Sun tutorial 4 . The chosen personalization example focuses on link adaptation; other adaptation aspects are covered in [9].

The tutorial starts with an overview of available lectures, as represented by the Overview state, which summarizes the available lectures in the tutorial, as specified by the Summary value in the LearningPresentation tagged value. It also presents the high level tutorial steps (Tutorial value in the CourseStructure tagged value). Links from the overview point not only to the first section of the tutorial, but also to the other main sections; all these links, except the first one, are associated with guard conditions that check that the user has enough knowledge to jump directly to the respective lectures.

The next step from the Overview is a lecture on the Object Oriented Programming Concepts. This state is accessible without any prerequisite on background knowledge; it is a composite state, containing five steps, represented by four substates: What is an Object, What is a Message, What is a Class, Relations to Code, and Questions. The Relations to Code state also shows an entry procedure addressing content level adaptation. The procedure applies to a learning step about building programs; it states that if the current user does not have sufficient

\footnotetext{
${ }^{4}$ See http://java.sun.com/docs/books/tutorial/java/index.html
} 


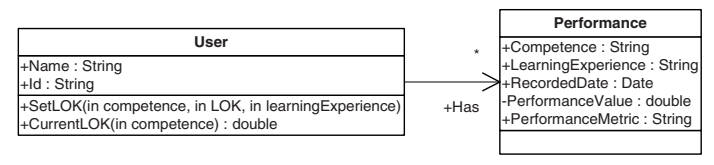

Fig. 4. A user model for the Java tutorial.

knowledge on basic concepts about object-oriented programming procedures, then learning content on procedures will be added.

The next step from the Object Oriented Programming Concepts is the composite state Language Basics. The transition between the two states features a next event and a guard. The guard specifies a link level adaptation rule, saying that the link is recommended when current user level of knowledge is greater then zero. The other learning steps modelled in the state diagram can be interpreted similarly.

The personalization specification within state diagrams is based on the user model depicted in Figure 4 It is inspired by the LTSC IEEE 1484.2 Learner Model WG Standard proposal for public and private information (PAPI) for learne 56. The user model is composed of the classes User and Performance, plus an association expressing that a learner can have several performance records based on the acquired LearningExperience and Competence.

The Performance class stores the user's level of knowledge about the concepts described by the tutorial. This value is the one used for determining if a transition into a new state is appropriate and must be suggested to a given user. For example, the following condition:

\section{[CurrentUser.CurrentLOK('Classes and Inheritance' ')>0]}

is a guard that in the state diagram determines wether a link can be followed between the Classes and Inheritance state and the Interfaces and Packages state, based on current user level of knowledge. The Performance class maintains as well the value of competence, recorded date, and metrics used to measure level of competence.

The User class provides operations to set and get the acquired level of knowledge or level of competence. These operations are used in guards and actions for adaptivity rules, and for updating learner profile. For example, in the state diagram of Figure 3 the user level of knowledge about "Classes and Inheritance" can be acquired either in the Object Oriented Programming Concepts lecture or in the Classes and Inheritance lecture. Exit procedures of these states indeed contain similar update operations, as the one which follows:

CurrentUser.SetLOK ('Classes and Inheritance', ,0.2, Content).

\footnotetext{
5 http://ltsc.ieee.org/archive/harvested-2003-10/working_groups/wg2.zip

${ }^{6}$ For a more detailed learner profile, used e.g. in EU/IST Elena (http://www.elena-project.org), the reader is referred to the learner RDF bindings web site at http://www. learninglab.de/ dolog/learnerrdfbindings/.
} 
In UML-Guide, state diagrams are used as input for visualizing navigation maps, whose structure is made of documents (nodes), composite nodes (folders), links (arrows), and parallel regions (dashed boxes). State diagrams are edited by means of the commercial tool Poseidon 7). The navigation map is then generated through a transformation method [9], whose input is the state diagram encoded in XMI (as produced by Poseidon), and whose output is the map.

\section{Integration of WebML and UML-Guide}

The integration of WebML with UML-Guide proposed in this paper aims at composing a generic "vertical e-learning" WebML application with a UML-Guide that is focused on a specific learning goal. We offer to the users of the composite system the standard, WebML-generated interface of the vertical, populated by content spawning a large body of knowledge; but we also offer to the focused learners a guide, available on an interface that can be opened "aside" the main one, and that points to pages and contents published by the WebML-generated interface, according to a specific learning objective and user experience.

The integration is loose and preserves the distinctive features of the two systems. In particular, some nodes and links in a UML-Guide state diagram point to content which is managed in the WebML e-learning vertical; therefore, the integration of UML-Guide with WebML requires UML-Guide adopting WebML concepts, such as page identifiers and content identifiers. In this way, concepts used as state names or as tagged values within UML-Guide are mapped to learning resources stored in the database generated from the WebML data model.

In the resulting application, the user-specific adaptation occurs in UML-Guide. This separation of concerns represents an extreme solution, as it is possible to support personalization [5] and adaptation [2] directly in WebML. However, the proposed solution is an example of how client-side computations, specified at high-level in UML, can integrate WebML-designed solutions. As such, this experiment can be replicated for many other applications and the focus on UML-Guide can pursue different objectives.

Figure 5 describes the system architecture. The high-level WebML and UML-Guide specifications are mapped into XML-based internal representations, respectively built by the Code Generator component of WebRatio [4] and by the XMI [13] Generator of Poseidon.

The WebML run-time component runs JSP templates (also embedding SQL), and uses XSL style sheets for building the application's presentation. The XMI representation of a UML-Guide drives a run-time adaptation engine, written in XSLT, which dynamically changes the content of the profile variables and produces the UML-Guide user interface. The WebML and UML-Guide interfaces are then composed and presented to the user.

In this architecture, the main integration issue is concerned with the generation of WebML links "pointing" to the WebML-controlled portion of the application, to be addressed while building the UML-Guide interface. WebML links take the format:

${ }^{7}$ http://www.gentleware.com/ 


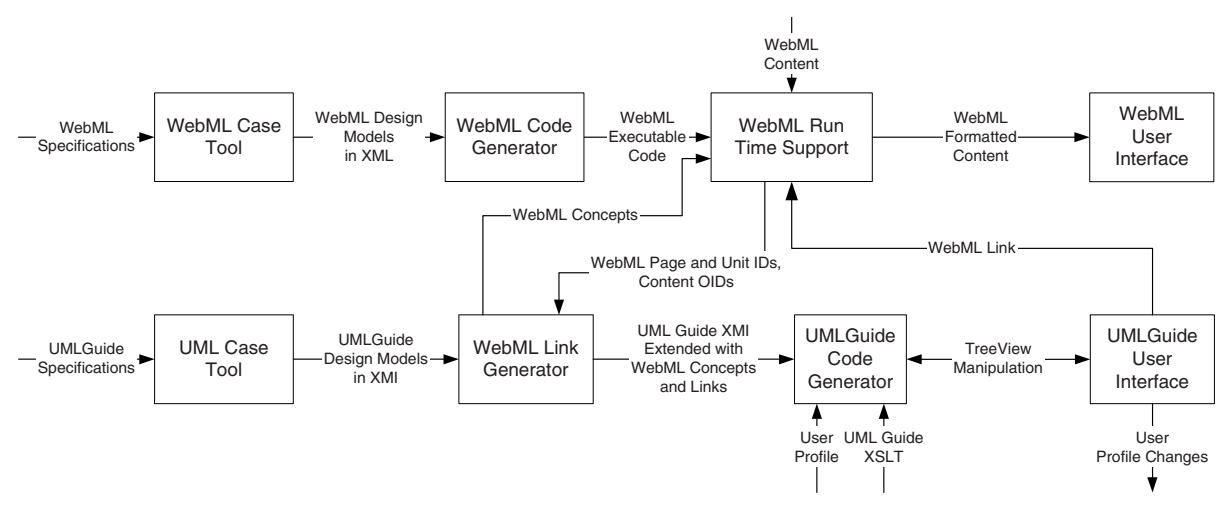

Fig. 5. Architecture of the composed system.

ApplicationURL/page_identifier.do?ParameterList

where page_identifier denotes a WebML page and ParameterList is a list of tag-value pairs, in the form entity_id.attribute=parameter. Thus, UML-Guide state diagrams must be extended with tagged values to be used as pointers to WebML concepts.

Figure 6 depicts an excerpt of state diagram extended with tagged values for WebML concepts, needed for computing WebML links. This work must be performed by UMLGuide designers, typically in the course of the transformations required for "implementing" UML-Guides starting from their high-level descriptions (as illustrated in Figure 3).

For instance, Object Oriented Programming Concepts is a lecture. The corresponding page name is LectureModules from WebML hypertext model. The entity used to store lectures in the WebML data model is LO. The title used as an attribute to identify the lecture is the same as the state name. Entry and exit actions are transformed if they send parameters in WebML links, as it is in the case of Relations To Code (where the parameter of the show method is replaced by specific WebML parameter \&LO. Title=Procedures). It is worth noting that, although in our example tagged values for page and entity names are constant values, in more complex cases they can be specified as well as parametric selectors, so as to automatically retrieve their values from the WebML XML specification based on specific conditions. Also, more tagged values can be needed, for example for identifying content units IDs, in situations where the selection of pages is based upon the content units they include.

Queries for retrieving OID's of the WebML concepts and content are submitted through a specifically designed interface to the WebML run-time components. The interface consists of the two functions GetWebMLConcept (Type, Name) and GetWebMLRecordOID(Entity, Attribute, Value).

\section{Generation of the Integrated E-learning Application}

Figure 7 presents the user interface of the integrated application. The UML-Guide generated map, obtained as a transformation of the UML state diagram depicted in Figure 3 


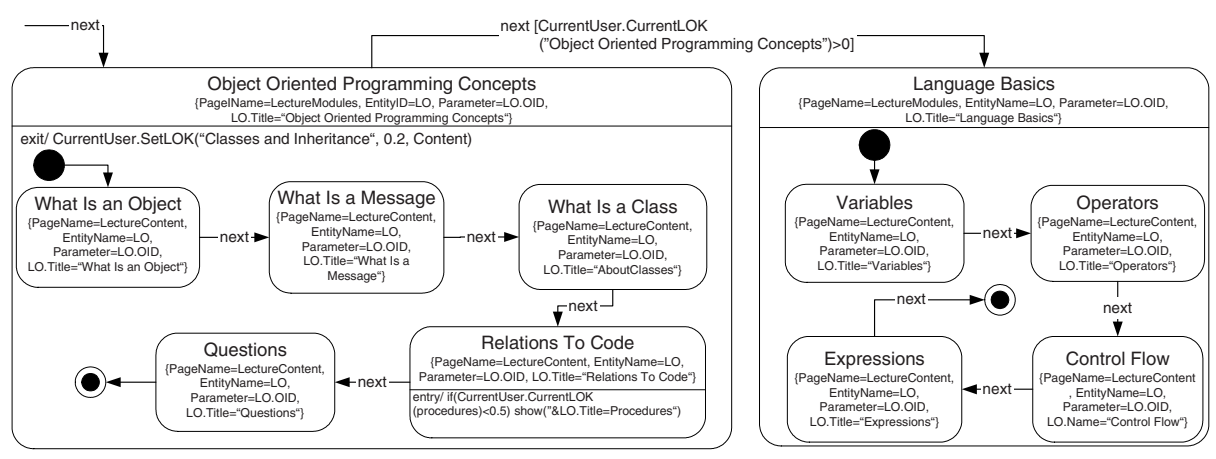

Fig. 6. Excerpt of the UML-Guide state diagram extended with tagged values representing WebML concepts.

is on the left; the WebML application, generated from the specification of Figure 2, is on the right. While the WebML application has an arbitrary interface, which depends on content composition within pages and on the adopted presentation style, the UML-Guide interface has a given structure that includes the following elements:

- Folder symbol-represents a composite information fragment composed by other (simple or composite) information fragments and links. The composition is visually represented by the plus/minus symbol, for showing/hiding enclosed items, and by the left hand indent of enclosed items. A content can be associated to each symbol.

- Dashed box symbol-represents a composite information fragment, which has to be presented concurrently with other composite information fragments (the dashed boxes) depicted on the same level.

- Document symbol-represents a simple information fragment; only links can be nested under it.

- Arrow symbol-represents a link to another composite or simple information fragment; the arrow symbols can be nested under the folder when they represent different alternatives of suggested links starting from a particular document. Each arrow is associated with a content and a name of the corresponding target node. Also, the "/next" string is added to names of arrows representing guidance to the next fragment according to the course sequence.

- Grayed background of nodes-represents the currently presented node, i.e., the position reached by a user in the navigation map.

Presentation for the adaptive navigation support depends on the generator settings. For example, according to the traffic light metaphor, adaptive recommendations may be represented through different colors (green for nodes appropriate with respect to the current state of the user profile, red for not appropriate nodes, yellow for other situations - e.g. a node that has been already visited). Also, other metaphors might show, hide, or sort the nodes.

Profile records are maintained at the client side. When users begin a new session, their profile is initialized from a client-side XML-based database. The navigation map is 


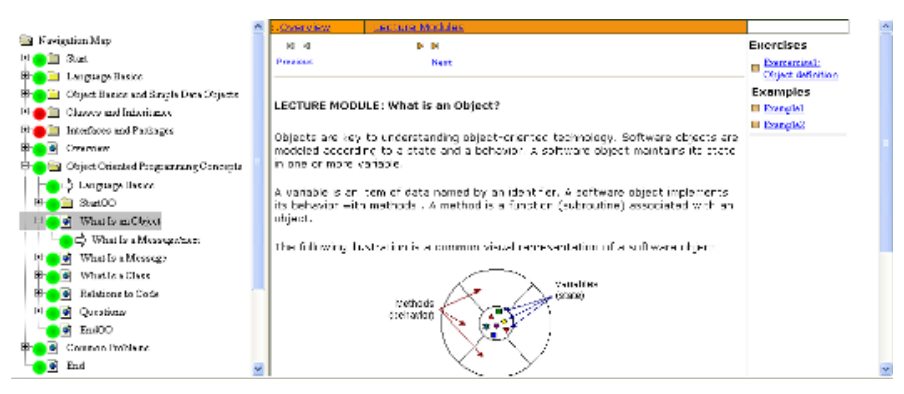

Fig. 7. Visualization of the navigation graph for the Java e-lecture.

manipulated at the client side as well. Javascript is used to implement the user interface control and user profile manipulation. The events generated by user actions on the user interface invoke profile adaptation actions, which possibly process and add new values to the user profile. They also trigger regeneration of the navigation map, according to the newly computed values.

The navigation map responds to changes in user profile by changing recommendation annotations (e.g., changing colors of nodes or showing/hiding nodes). When specific requirements, for example those set by conditions in entry actions of states, are met, the WebML vertical adapts delivered content based on additional parameters that UMLGuide is able to send to the server-side application.

\section{Related Work}

Model-driven development of Web applications has been intensely investigated during last years 610,1117]. WebML has been proposed for the model-driven design of "dataintensive" Web applications. Its distinguishing feature is that the proposed design method is also supported by XML- and Java-based technologies, which enable the automatic generation of the application code [4].

During last years, some approaches have been proposed for extending traditional development methods by means of features enabling one-to-one personalization 115 17]. The aim is to customize the applications contents and the hypertext topology and presentation with respect to user preferences and needs, so as to offer an alternative to the traditional "one-size-fits-all" static approach in the development of Web systems [1]. Some proposals cover the adaptivity of Web applications with respect to some other dimensions characterizing the context of use (see [14] for a complete survey). WebML also offers some constructs for personalization. In particular, the application data schema can be extended through some entities representing user profiles and user access rights over the application content [5]. Recently, WebML has also been extended with some primitives for modelling context-aware applications, i.e., mobile, personalized applications that are also able to adapt to the current situation of use [2]. However, WebML, as well as the majority of Web personalization and adaptivity approaches so far proposed, manages personalization at server-side, and does not offer the alternative of managing user profiles 
and personalization policies at client side. Conversely, the UML-Guide approach establishes model-driven design for adaptive applications, by considering link level adaptation and content level adaptation at the client side, where adaptation is computed according to the UML design specifications. First, requirements are modelled as variation points with mandatory and optional features in application domain models [7]. Guard logical expressions and adaptivity actions are used in navigation specifications [9]. A rule based approach has been also employed in more open environment based on semantic web models [8].

\section{Conclusions and Further Work}

This paper has shown the integration between WebML and UML-Guide; the proposed approach demonstrates that server-side and client-side technologies can coexist and that it is possible, for both of them, to use model-driven code generation techniques starting from high-level requirements, expressed in graphical form. The proposed application scenario augments an "e-learning" vertical so as to make it adaptable and personalisable.

In our experiments, we moved especially user dependent functionality to the client side which allowed us to leave control over sensitive user data to the user. Users can decide on their own which information will be disclosed and which they will deny access to. On the other hand, this increases requirements for client-side tools to be able to interpret a database with information about a user and process it for purpose of adaptation and personalization. As client machines are usually less powerful, this might result in some lacks of performance. We will further investigate and experiment with the approach proposed to find a good balance between client-side and server-side processing.

We regard this work as the first step of a deeper methodological inspection of the interactions between UML and WebML, and more specifically of the possibility of using state diagrams, which best represent the modeling of dynamic interfaces, for collecting the requirements that can naturally evolve into WebML specifications. The experiments described in this paper, and specifically the mechanisms for rendering state transitions as WebML links, will be extended and reused. In this paper we showed an integration of the two methods on an application where the state diagram is used to model interaction over information from one class. As a part of the deeper investigation, more complex applications will be studied where interaction between objects of several classes implies a need to use other behavioral techniques (collaboration and sequence diagrams) together with state diagrams.

We are also planning an extension of the WebML CASE tool and of UML-Guide for providing automatic support to the integration of the two methods.

\section{References}

1. P. Brusilovsky. Adaptive Hypermedia. User Modeling and User-Adapted Interaction, 11(12):87-100, 2001.

2. S. Ceri, F. Daniel, and M. Matera. Extending WebML for Modeling Multi-Channel ContextAware Web Applications. In Proceedings of WISE-MMIS'03 Workshop, Rome, Italy, December 2003, pages 615-626. IEEE Computer Society, 2003. 
3. S. Ceri, P. Fraternali, A. Bongio, M. Brambilla, S. Comai, and M. Matera. Designing DataIntensive Web Applications. Morgan Kauffmann, 2002.

4. S. Ceri, P. Fraternali, et Al. Architectural Issues and Solutions in the Development of DataIntensive Web Applications. In Proc. of CIDR'03, Asilomar, CA, USA, 2003.

5. S. Ceri, P. Fraternali, and S. Paraboschi. Data-Driven One-To-One Web Site Generation for Data-Intensive Web Applications. In Proceedings of VLDB'99, September 1999, Edinburgh, UK, pages 615-626. IEEE Computer Society, 1999.

6. J. Conallen. Building Web Applications with UML. Object Technology Series. Addison Wesley, 2000.

7. P. Dolog and M. Bieliková. Towards Variability Modelling for Reuse in Hypermedia Engineering. In Y. Manolopoulos and P. Návrat, eds., Proc. of ADBIS 2002, LNCS, vol. 2435, pages 388-400. Springer, 2002.

8. P. Dolog, N. Henze, W. Nejdl, and M. Sintek. Personalization in Distributed e-Learning Environments. In Proc. of WWW2004, May 2004. To appear.

9. P. Dolog and W. Nejdl. Using UML and XMI for Generating Adaptive Navigation Sequences in Web-Based Systems. In P. Stevens, J. Whittle, and G. Booch, eds., Proc. of UML 2003-The Unified Modeling Language, LNCS, vol. 2863, pages 205-219. Springer, 2003.

10. P. Fraternali. Tools and Approaches for Developing Data-Intensive Web applications: A survey. ACM Computing Surveys, 31(3):227-263, September 1999.

11. F. Garzotto, P. Paolini, and D. Schwabe. HDM-a Model-Based Approach to Hypertext Application Design. ACM Transactions on Information Systems, 11(1):1-26, January 1993.

12. O. M. Group. OMG Unified Modelling Language Specification, version 1.3, Mar. 2000. Available at http://www.omg.org/. Accessed on June 1, 2001.

13. O. M. Group. OMG XML Metadata Interchange (XMI) Specification, ersion 1.1, Nov. 2000. Available at http://www.omg.org/. Accessed on June 1, 2002.

14. G. Kappel, B. Proll, W. Retschitzegger, and W. Schwinger. Customization for Ubiquitous Web Applications: a Comparison of Approaches. International Journal of Web Engineering and Technology, 11, January 2003.

15. N. Koch and M. Wirsing. The Munich Reference Model for Adaptive Hypermedia Applications. In P. D. Bra, P. Brusilovsky, and R. Conejo, eds., Proc. of AH2002-Adaptive Hypermedia and Adaptive Web-Based Systems, LNCS, vol. 2347. Springer, 2002.

16. K. Marriott, B. Meyer, and L. Tardif. Fast and Efficient Client-Side Adaptivity for SVG. In Proc. of WWW 2002, May 2002, Honolulu, Hawaii, USA, pages 496-507. ACM Press, 2002.

17. D. Schwabe, R. Guimaraes, and G. Rossi. Cohesive Design of Personalized Web Applications. IEEE Internet Computing, 6(2):34-43, March-April 2002.

18. G. South, A. Lenaghan, and R. Malyan. Using Reflection for Service Adaptation in Mobile Clients. Technical report, Kingston University-UK, 2000. 